\title{
Jamming Threshold of Dry Fine Powders
}

\author{
J. M. Valverde, M. A. S. Quintanilla, and A. Castellanos \\ Departamento de Electronica y Electromagnetismo, Universidad de Sevilla, Avenida Reina Mercedes s/n, 41012 Sevilla, Spain
}

(Received 12 November 2003; published 24 June 2004)

\begin{abstract}
We report a novel experimental study on the jamming transition of dry fine powders with controlled attractive energy and particle size. Like in attractive colloids dry fine particles experience diffusionlimited clustering in the fluidlike regime. At the jamming threshold fractal clusters crowd in a metastable state at volume fractions depending on attractive energy and close to the volume fraction of hard nonattractive spheres at jamming. Near the phase transition the stress-(volume fraction) relationship can be fitted to a critical-like functional form for a small range of applied stresses $\sigma \sim$ $\left(\phi-\phi_{J}\right)^{\beta}$ as measured on foams, emulsions, and colloidal systems and predicted by numerical simulations on hard spheres.
\end{abstract}

DOI: 10.1103/PhysRevLett.92.258303

PACS numbers: 83.80.Fg, 81.20.Ev, 47.55.Kf

Supercooled liquids, granular systems, and colloidal suspensions are systems that display a nonequilibrium kinetic transition from a fluidlike to a solidlike jammed regime [1]. At jamming the constituent particles are suddenly arrested in a metastable static state forming a solid disordered network that spans the system. The jamming transition has been described by a phase diagram parametrized by interparticle attractive energy $U$, temperature $T$, particle volume fraction $\phi$, and applied stress $\sigma[2,3]$. For example, granular systems jam when they are compressed or shear stress is lowered, a liquid jams when it is cooled, and colloid particles gelate with increasing $U$. Light scattering experiments suggest a link between the jamming transitions for suspensions of hard (nonattractive) spheres $\left(U / K_{B} T=0\right.$, where $K_{B} T$ is the thermal energy) and for suspensions of attractive particles $\left(U / K_{B} T>0\right)$ [4]. While the kinetic arrest is driven by crowding of single particles in the absence of attractive forces, for attractive suspended particles jamming is driven by the crowding of fractal clusters. Suspensions of nonattractive hard spheres jam at $\phi=\phi_{J} \approx$ 0.56-0.59, which is comparable to the random loose packing (RLP) of nonattractive hard spheres at the limit of zero gravitational force $\left(\phi_{\mathrm{RLP}} \simeq 0.56\right)$ but is well below the random close packing $(\mathrm{RCP})$ limit $\left(\phi_{\mathrm{RCP}} \simeq 0.64\right)[5]$. On the other side, jamming of strongly attractive suspended particles takes place at $\phi_{J}=\phi_{J}(U)$. In the limit $U / k_{B} T \gg 1$ fractal clusters crowd by a diffusion-limited cluster-cluster aggregation process (DLCA [6]). Since the density of this fractal structure decreases as it grows the system can form a gel at arbitrary small $\phi\left(\phi_{J} \ll 1\right)$ [4]. For $U / k_{B} T>\sim 1$, Segre et al. [4] found a phase transition boundary that reminds a thermally activated process. A similar functional form was measured by Prasad et al. [1]. Yet the details of jamming in this intermediate regime are far from being fully understood [1].

Simulations on frictionless spheres suggest that the behavior of the jammed system in the vicinity of the transition could be understood as a perturbation around the "most disordered" jammed state $(J)$ (see [7] for a discussion). Among other aspects numerical simulations show that the applied stress $\sigma$ on the weak solid network follows a power-law scaling $\sigma \sim\left(\phi-\phi_{J}\right)^{\beta}$ close to the transition, typical of an ordinary critical behavior. The critical exponent is found to depend on the kind of interparticle interaction, $\beta \simeq 1.5$, for Hertzian-type interparticle contact [7,8] and $\beta \simeq 1$ for harmonic contact [7]. This critical-like behavior seems to be a universal characteristic of jammed systems and it is also found in attractive colloids, concentrated emulsions, and foams $[3,9,10]$.

Even though fine powders share common aspects with noncohesive granular materials and with attractive colloids, they must be considered as a separate system. Like noncohesive granular materials, but unlike attractive colloids, fine powders are athermal, yet attractive forces between fine particles are relevant as for attractive colloids. For this reason it is interesting to study the jamming transition in fine powders in order to extend further its commonality and thus generalize the phase diagram as a unifying description. In this Letter we probe experimentally the jamming transition for fine powders and look for the effects of the attractive energy and particle size.

The powders used are xerographic toners based on polystyrene (particle density $\rho_{p}=1.135 \mathrm{~g} / \mathrm{cm}^{3}$ ). Particles are produced by an attrition process followed by size classification using a cyclone separator apparatus [11]. In this way powders of volume average particle diameter $d_{p}$ ranging from 19.1 to $7.8 \mu \mathrm{m}$ are obtained. For uncharged dry particles the main source of interparticle attraction arises from the short ranged van der Waals interaction that yields $U \simeq A d_{a} /\left(24 z_{0}\right)$ between two particles at contact [11]. Here $A$ is the Hamaker constant $(A \sim$ $\left.10^{-19} \mathrm{~J}\right), d_{a}$ is the typical size of the particle surface asperities (typically $d_{a} \sim 0.2 \mu \mathrm{m}$ in most powders [11]), and $z_{0}$ is the shortest distance between two neighboring particles. At $z_{0}$ the net interparticle force $F \simeq A d_{a} /\left(24 z_{0}^{2}\right)$ 
is attractive and reaches a maximum (most investigators obtain $z_{0} \simeq 4 \AA$, independent of $\phi$ [11]). For loaded contacts however the interparticle adhesive force depends on the load force and decreases with the contact hardness [12]. Nevertheless the stress on the particles is $\simeq 0$ at the jamming threshold and therefore the van der Waals equation is a good estimation of the interparticle energy. The powders produced are blended with fumed silica nanoparticles $(\simeq 7 \mathrm{~nm}$ particle size). Scanning electron microscopy (SEM) micrographs show that silica nanoparticles are agglomerated with estimated agglomerate diameters from 40 to $60 \mathrm{~nm}$ [13]. These agglomerates coat uniformly the toner particle surface [13] (see Fig. 2, inset). Thus the typical size of surface asperities at contact can be decreased from $d_{a} \sim 0.2 \mu \mathrm{m}$ to $d_{a} \sim 40 \mathrm{~nm}$ if a high surface additive coverage is used. The control of van der Waals force by tailored surface roughness and, in particular, by silica coating has been measured elsewhere by atomic force microscopy [13]. It can be roughly estimated that the transition from polymer-polymer to polymer-silica contacts should take place at $10 \%$ of surface area coverage (SAC) of additive (theoretical value assuming perfectly spherical toner particles) whereas the transition from polymer-silica to silica-silica contacts should occur at $80 \%$ SAC [14]. Accordingly flow properties experience a marked variation when SAC is varied within this range [14]. In this Letter the influence of $U$ on jamming will be explored by testing toner samples with $32 \%$ and $64 \%$ SAC. Because of the uniform coating we may assume on average a continuous linear decrease of the typical asperity size and estimate a relative decrease of interparticle energy $\sim 50 \%$ when the $\mathrm{SAC}$ is reduced from $64 \%$ to $32 \%$. Commercially available toner (low cohesive) Canon CLC700 $\left(d_{p} \simeq 8.5 \mu \mathrm{m}\right)$ and nonattractive monosized poly(methyl methacrylate) (PMMA) beads $\left(d_{p}=200 \mu \mathrm{m}\right)$ of approximately the same density than toner particles have been also tested for comparison.

Microgravity is the ideal environment to investigate the intrinsic properties of jamming since sedimentation due to gravity, which obscures the natural behavior of the system, is avoided. An alternative technique is fluidization, which allows us to remove weight and bypass jamming in a highly controllable fashion. In this technique powder samples are supported on a porous plate in a vertically oriented cylindrical vessel. A controlled flow of dry nitrogen is introduced into the lower part of the vessel while the gas pressure drop across the bed $\Delta p$ is measured. The average $\phi$ is obtained from the bed height measured by an ultrasonic pulser receiver at the top of the vessel. As a previous step we impose a gas flow sufficiently large to drive the bed into the bubbling regime, in which the powder loses memory of its previous history [15]. Then the gas flow is decreased and $\Delta p$ and $\phi$ measured. From $\Delta p$ the normal consolidation stress $\sigma=$ $W-\Delta p$ is obtained.

Figure 1 displays results of $\phi$ as the gas flow is decreased for the noncohesive PMMA beads. Data obtained

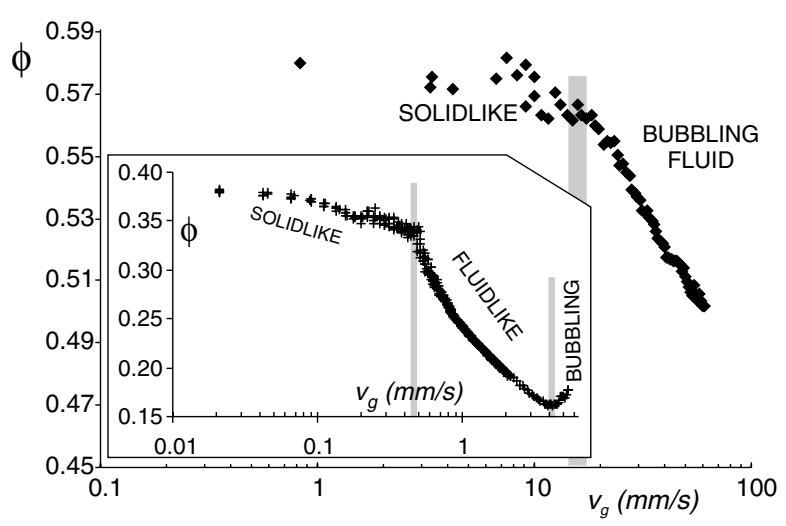

FIG. 1. Particle volume fraction of noncohesive PMMA beads $\left(d_{p}=200 \mu \mathrm{m}\right)$ in the fluidized bed as a function of the gas velocity. The inset shows the data for a cohesive powder $\left(d_{p}=\right.$ $11.8 \mu \mathrm{m}, 64 \% \mathrm{SAC}$ ). The jamming transitions are indicated by vertical bars.

for a fine powder are shown in the inset. According to the large size of the PMMA particles this material is a typical B powder of the Geldart's scheme, bubbling immediately on moderate expansion [16]. Thus there is a direct transition from the bubbling fluidlike regime to the solidlike regime [16]. Note that at jamming $\phi_{J} \simeq 0.56 \simeq \phi_{\mathrm{RLP}}$ as we might have expected. As seen in the Fig. 1 inset, and due to the effectiveness of attractive forces in hindering rearrangement, the transition to the solidlike state in the fine powder is observed at smaller $\phi\left(\phi_{J} \simeq 0.33\right)$. As the gas flow is further decreased below the jamming threshold the strong interparticle interaction produces a change of behavior which is more marked in the presence of attractive forces.

A feature that distinguishes fluidized beds of fine powders from fluidized beds of noncohesive granular materials is the existence in the former of a nonbubbling fluidlike regime preceding the onset of jamming [17]. In this fluidlike regime primary particles are clustered due to attractive forces and clusters undergo an intense hydrodynamic interaction with the gas that prevents the development of macroscopic gas bubbles [18]. The number of particles per cluster $(N)$ and the ratio of cluster size to $d_{p}(k)$ are obtained by means of sedimentation experiments (see Ref. [19] for details). In all the ranges of $d_{p}$ and SAC investigated the fractal dimension of the clusters $D=\ln N / \ln k$ approaches the diffusion-limited aggregation (DLA [6]) theoretical value $D=2.5$. Growth of DLA clusters in the fluidlike regime is the result of the combined action of the effective fast diffusive process followed by the particles and the strong interparticle attraction. The accretion of particles onto clusters stops when the drag force balances intercluster attraction which limits the final cluster size. (It must be warned that we only observe the fractal scaling over finite length scales and therefore we cannot ascertain in the strict sense of the word that our clusters are really fractal. In a recent investigation however Nam et al. [20] use our technique 
and obtain fluidized DLA clusters of $\sim 10^{11}$ nanoparticles.) A nondimensional number that determines the size of the clusters is the ratio of the interparticle attractive force to particle weight (granular Bond number $\mathrm{Bo}_{g}$ ) [19] which is typically $\gg 1$ for fine powders. For example, assuming an interparticle contact between silica agglomerates $\left(d_{a} \simeq 40 \mathrm{~nm}\right), B o_{g}$ can be estimated to increase from $\sim 40$ to $\sim 600$ when $d_{p}$ is decreased from $19.1 \mu \mathrm{m}$ to $d_{p}=7.8 \mu \mathrm{m}$. Even though $B o_{g} \gg 1$, especially for the smaller particles, the granular Bond number of the clusters viewed as effective particles $\left(B o_{g}^{*}=\right.$ $B o_{g} / N$ ) is only slightly above 1 and quite independent of particle size. A Bond number of order 1 is roughly the limit that distinguishes free flowing individual grains from aggregative cohesive powders (see [19] for further discussion).

In the fluidlike regime the bed does not have a mechanical yield stress and the powder weight per unit area $W$ is completely balanced by the gas pressure drop. As the gas flow is further decreased there comes a critical point at which clusters are jammed in enduring positions, the structure gets a solidlike appearance, develops a yield stress, and the self-diffusion coefficient vanishes [17]. At the jamming threshold enduring cluster contacts support part of the structure and thus $\Delta p$ crosses below the weight. The normal consolidation stress $\sigma=W-\Delta p$ becomes then positive and increases as the gas velocity is further decreased. We observe systematically that $\phi$ at jamming $\left(\phi_{J}\right)$ does not depend on the sample size since, unlike in colloidal gels, the crowding of clusters is not the result of a DLCA process (otherwise $\phi_{J}$ should decrease as the sample grows in size). On the contrary clusters crowd near jamming with low sticking probabilities as corresponds to weakly attractive effective particles. The fluid-to-solid transition is extremely sensitive to the applied stress as can be derived from the fluctuations of the local concentration of particles. Near jamming we see the nucleation of transient (avalanchelike) fluid structures driving the relaxation time of the system to diverge (see Fig. 2 of Ref. [21]). A similar behavior has been reported for the gelation transition of colloids, the colloidal glass transition [4], and the melting transition of foams [9].

In the Fig. 2 inset we plot $\phi$ as a function of $\sigma$ nearby jamming for toner Canon CLC700. Jamming, characterized by the development of a finite stress $(\sigma>0)$, takes place for $\phi \simeq \phi_{J} \simeq 0.28$. The main graph of Fig. 2 shows that, in the range $\sigma<\sigma_{Y} \simeq 15 \mathrm{~Pa}$, the data fit pretty well to a power-law functional form $\sigma \propto\left(\phi-\phi_{J}\right)^{\beta}$, previously recognized in foams and emulsions [10], in attractive colloids [3], and predicted by simulations of hard sphere systems $[7,8]$. To our knowledge ours are the first experimental results on dry granular materials. Note the small value of the critical exponent $(\beta \sim 0.8)$ as compared with that obtained in simulations of hard noncohesive spheres interacting via Hertz potential $(\beta \simeq 1.5)$. Another characteristic is that for normal stresses $\sigma>$ $\sigma_{Y} \simeq 15 \mathrm{~Pa}$ the experimental data deviate clearly from

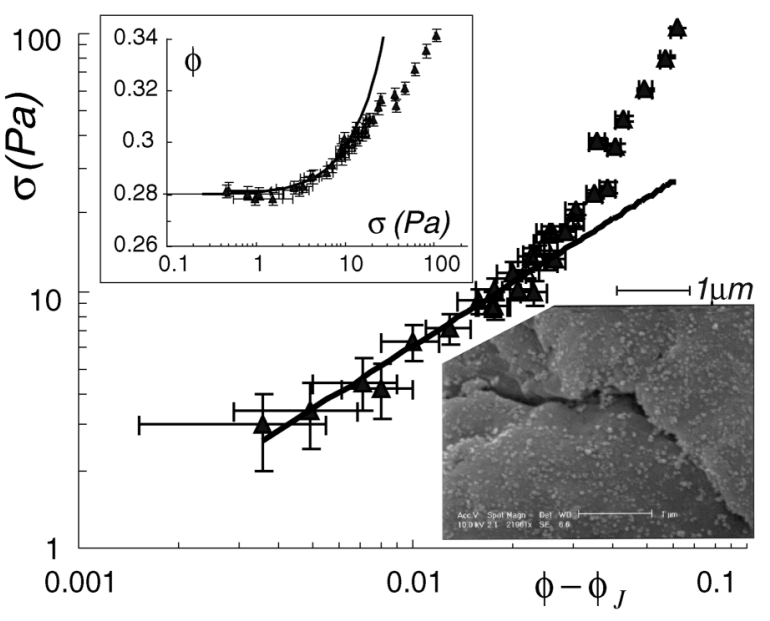

FIG. 2. Normal stress as a function of the increment of the particle volume fraction beyond the jamming threshold $(\sigma>$ 0 ) for Canon CLC700 powder. Insets: (top) $\phi$ as a function of $\sigma$ showing $\phi_{J} \simeq 0.28$; (bottom) SEM micrograph of a toner particle surface with $64 \%$ SAC. The continuous line corresponds to the power law $\sigma \simeq 271\left(\phi-\phi_{J}\right)^{0.824} \mathrm{~Pa}$ that is fitted to the data in the range $\sigma<\simeq 15 \mathrm{~Pa}$.

the power-law trend. Both results suggest a special role of the attractive interaction that will be discussed below.

In Fig. 3 results for powders with common SAC 32\% (i.e., the same $U$ ) but with different $d_{p}$ are presented. It is seen that $\phi_{J}$ decreases as $d_{p}$ is decreased while $U$ is kept constant. In general the ability of particles to rearrange themselves at a given normal stress $\sigma$ worsens as $d_{p}$ is decreased. The results show however that the critical exponent is similar for all the powders $(\beta \sim 0.8)$ which suggests a common behavior. To test our hypothesis that clusters would behave as weakly attractive particles near jamming we plot in Fig. 4 the cluster volume fraction $\phi^{*}=\phi k^{3} / N$ against the normal stress. It is observed that within the experimental scatter the data are approximately scaled onto a single master curve and $\phi_{J}^{*} \simeq 0.51$

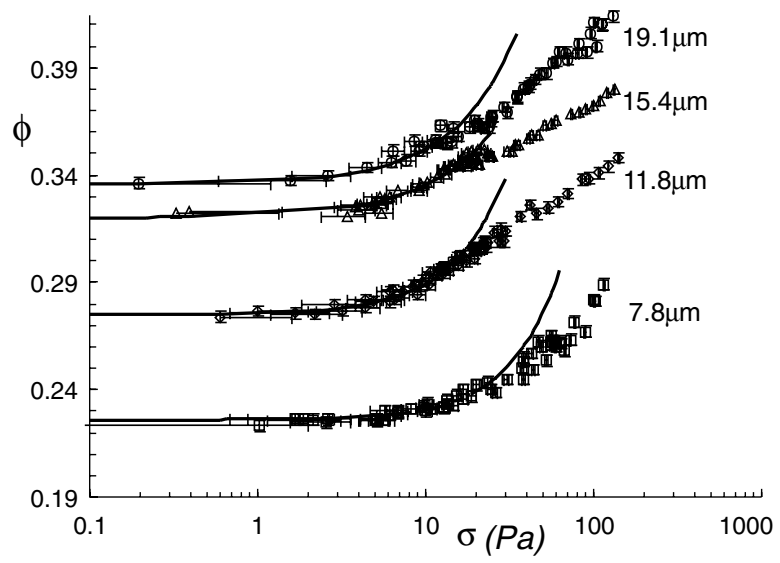

FIG. 3. Particle volume fraction as a function of the normal stress for a set of experimental toners with varying particle size (indicated) and the same $U(32 \% \mathrm{SAC})$. The continuous lines represent the power law $\sigma=\alpha\left(\phi-\phi_{J}\right)^{\beta}$ that is fitted to the data in the range $\sigma<\simeq 20 \mathrm{~Pa}$. 


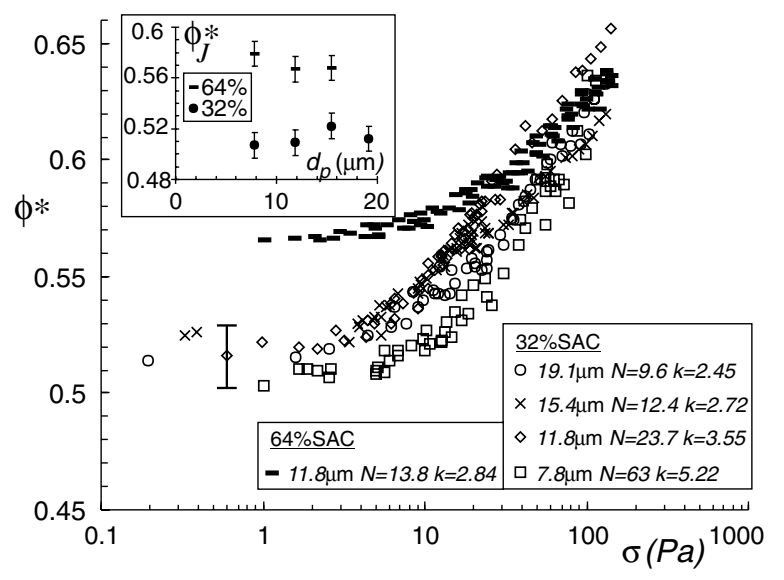

FIG. 4. Cluster volume fraction $\phi^{*}=\phi k^{3} / N$ as a function of the normal stress for the set of experimental toners of Fig. 3. Also plotted are data for the $11.8 \mu \mathrm{m}$ particle size, $64 \%$ SAC experimental toner. The error bar is the typical indeterminacy in $\phi^{*}$. The inset shows the cluster volume fraction at the jamming transition $\phi_{J}^{*}$ as a function of particle size.

irrespective of $d_{p}$ for a given $U$. In fact the size of clusters is more or less independent of $d_{p}$ for constant $U$. Within the range of our experimental materials our results indicate that the clusters of the different powders might behave as effective spheres with the same size and attractive energy and only slightly different densities $\left(\rho_{c}=\right.$ $\left.N / k^{3} \rho_{p}\right)$. As the internal particle volume fraction of the clusters $\left(\phi_{\text {int }}=N / k^{3} \approx 1 / \sqrt{k}\right)$ decreases with $k$, it turns out that $\phi_{J}$ decreases as the primary particle size is decreased and $k$ increases (as seen in Fig. 3). At the jamming threshold we find $\phi_{J}^{*} \sim 0.54$ for Canon CLC700 and $\phi_{J}^{*} \simeq 0.57$ for the set of experimental toners with $64 \%$ SAC (see Fig. 4 inset). Interestingly $\phi_{J}^{*}$ for these clusters of decreased attractive energy is quite similar to the values of $\phi_{J}^{*}$ typically reported for suspensions of noncohesive hard spheres [5].

In light of our results the physical mechanism involved on jamming can be rationalized as follows: At the jamming transition clusters would pack in the loosest random packing in the absence of gravity $\phi_{J}^{*}$ that depends on $U$. Likely clusters rearrange at low stresses $\left(\sigma<\sim \sigma_{Y}\right)$ by rolling, as seen by Blum et al. in their study of the impact of fractal aggregates onto solid targets [22], paralleling the behavior of slightly attractive spheres. Fractal clusters are easily deformable and therefore they should behave similarly to soft spheres rather than to hard spheres. Accordingly we obtain critical exponent $\beta$ close to 1 as it is obtained for soft particle granular systems such as foams and emulsions $[9,10]$. For $\sigma>\sim \sigma_{Y}$ the volume fraction increase is clearly below the power-law prediction. This might indicate a crossover to a more energy consuming physical mechanism of rearrangement, possibly consisting of the fracture of clusters that would have reached at $\sigma_{Y}$ their closest random volume fraction.
In conclusion, we have observed that for systems of dry, attractive, and athermal particles, jamming is driven by the kinetic arrest of DLA clusters. While the volume fraction of particles at jamming depends on particle size we have seen that the volume fraction of clusters $\phi_{J}^{*}$ is independent of primary particle size for a given $U$. The value of $\phi_{J}^{*}$ approaches the limit of noncohesive spheres as $U$ is decreased by means of surface additives. These results indicate that $\phi^{*}$ should be considered, rather than $\phi$, as the relevant parameter of a more general phase diagram. Despite the fact that jamming is clearly a nonequilibrium transition we find that in a small range of normal stresses the stress-(volume fraction) relationship fits to a critical-like functional form $\sigma \sim\left(\phi-\phi_{J}\right)^{\beta}$, which is a hallmark of the jamming transition observed in other systems.

This research has been supported by the Xerox Foundation and Spanish Ministerio de Ciencia y Tecnologia (BFM2003-01739).

[1] V. Prasad et al., Faraday Discuss. 123, 1 (2003).

[2] A. J. Liu and S. R. Nagel, Nature (London) 396, 21 (1998).

[3] V. Trappe et al., Nature (London) 411, 772 (2001).

[4] P. N. Segre et al., Phys. Rev. Lett. 86, 6042 (2001).

[5] P. N. Pusey and W. van Megen, Phys. Rev. Lett. 59, 2083 (1987).

[6] D. A. Weitz and M. Oliveria, Phys. Rev. Lett. 52, 1433 (1984).

[7] C. S. O'Hern et al., Phys. Rev. E 68, 011306 (2003).

[8] H. A. Makse, D. L. Johnson, and L. M. Schwartz, Phys. Rev. Lett. 84, 4160 (2000).

[9] D. J. Durian, Phys. Rev. Lett. 75, 4780 (1995).

[10] M. D. Lacasse et al., Phys. Rev. Lett. 76, 3448 (1996); T. G. Mason et al., Phys. Rev. E 56, 3150 (1997).

[11] K. Rietema, The Dynamics of Fine Powders (Elsevier, London, 1991).

[12] S. D. Mesarovic and K. L. Johnson, J. Mech. Phys. Solids 48, 2009 (2000).

[13] M. L. Ott and H. A. Mizes, Colloids Surf. A 87, 245 (1994); M. A.S. Quintanilla, A. Castellanos, and J. M. Valverde, Phys. Rev. E 64, 031301 (2001); H. Zhou, M. Gotzinger, and W. Peukert, Powder Technol. 135/ 136, 82 (2003).

[14] J. M. Valverde et al., Powder Technol. 97, 237 (1998).

[15] J. M. Valverde, A. Castellanos, and M. A. S. Quintanilla, Contemp. Phys. 44, 389 (2003).

[16] R. Jackson, The Dynamics of Fluidized Particles (Cambridge University Press, Cambridge, 2000).

[17] J. M. Valverde, A. Castellanos, and M. A. S. Quintanilla, Phys. Rev. Lett. 86, 3020 (2001).

[18] J. M. Valverde et al., Phys. Rev. E 67, 051305 (2003).

[19] A. Castellanos, J. M. Valverde, and M. A. S. Quintanilla, Phys. Rev. E 64, 041304 (2001).

[20] C. H. Nam et al., AIChE J. (to be published).

[21] J. M. Valverde et al., Phys. Rev. E 67, 016303 (2003).

[22] J. Blum and G. Wurm, Icarus 143, 138 (2000). 